\title{
Entropy Methods Application For Biosystems State Analysis*
}

\author{
Olga Berestneva ${ }^{1}$, Oxana Zharkova ${ }^{2}$, Yakov Pekker ${ }^{3}$, \\ Olga Marukhina ${ }^{4}$, Hatem M. A. Hassanin ${ }^{5}$, \\ ${ }^{1,5}$ Dept. of Applied Mathematics, ${ }^{2}$ Institute of Natural \\ Resources, ${ }^{3}$ Institute of Non-Destructive Testing, ${ }^{4}$ Dept. of \\ Optimization Control Systems \\ 1,2,3,4,5 National Research Tomsk Polytechnic University \\ Tomsk, Russia
}

\author{
Konstantin Sharopin ${ }^{6}$ \\ ${ }^{6}$ Dept. of Information Technologies \\ ${ }^{6}$ Moscow Humanitarian-Economic Institute \\ Moscow, Russia
}

\begin{abstract}
Adaptive possibilities biosystems appear only in real life conditions, namely in particular natural or artificial environment of an organism habitat. According to the authors, for the state of biosystems integrated assessment the promising approach is based on entropy methods for modeling complex systems.
\end{abstract}

Keywords-Adaptive possibilities biosystems, entropy methods, integrated assessment, state of biosystems, modeling complex systems.

\section{INTRODUCTION}

Entropy is a measure of the probability of staying in the present status of the system. It turned out that the entropy is one of the fundamental properties of any systems with probabilistic behavior been ensured vides new levels of understanding to encode information in the system analysis, linguistics, biology, image processing etc. Influence of external information in the system can be accessed through a change the entropy of the system status $[1,2]$.

Upon reaching the steady system status, the total entropy change can be considered approximately equal to zero, which corresponds to mutual compensation of all processes associated with the receipt, removal and transformation of substances, energy and information. Nobel laureate and Prigogine formulated the basic property of the steady status of open systems with fixed external parameters, the playback speed of entropy due to the occurrence of irreversible processes, is constant in time and the minimum value for $\mathrm{d} E$ / $\mathrm{d} t \rightarrow \min$.

Thus, according to theorem and Prigogine, the stationary status is characterized by minimal scattering entropy. For live systems, this situation can be summarized as: the maintenance of homeostasis requires a minimum consumption of energy, i.e. a healthy body is committed to working in the energy economy mode. With regard to diseases of the body, then it is associated with the additional energy costs for acquired compensation or with biological congenital defects and with an increase in entropy [2].

\section{ENTROPY TO EVALUATE STATUS OF THE HUMAN BODY}

The dynamical system may be multiple-stationary states

The research is conducted with financial support from The Russian Foundation for Basic Research, projects № 14-06-00026 and 15-07-08922. with differing levels of entropy playback $\mathrm{d} E k / \mathrm{d} t[1,2,3]$. From this point of view, condition of the body can be described as a set of energy levels, some of which are resistant (levels 1 and 4) other unstable (levels 2, 3, 5). If there are constantly acting external or internal disturbances can occur an abrupt transition from one status to another. Any replenishment is characterized by increased consumption of energy as: the body temperature rises, increases the rate of metabolism. Deviation from the steady status with minimal energy consumption causes the development of internal processes, seeking to restore the system back to level 1 . When the long-acting factors, the system can go to level 3, the socalled bifurcation point from which there are several possible outcomes: a return to a stable level 1, transition to another stable state of equilibrium 4 , characterized by new energylevel, or leapto a higher level of unstable - status 5 .

For the body, this corresponds to several levels of adaptation with respect to health or chronic disease with different levels of system performance. Acute illness corresponds to a steady status with high reproduction entropy, i.e. the type of uneconomical functioning of the body. According to the mathematical theory of catastrophes, in acute diseases is necessary to leap to transfer the body of «bad» steady status to «good». Thus using high doses of drugs. In the phase of fading exacerbation of disease, the role of small impacts, such as acupuncture and homeopathic remedies have a positive impact [1-3].

Entropy is an informative characteristic for status the body and can be used to assess its condition and treatment referral of patients. For example, the famous method of calculating the entropy of the gas discharge visualization (GRV) of biological objects and related software.

\section{ENTROPY FOR THE ANALYSIS OF CARDIAC RHYTHM}

More than 20 years of entropy estimates are used for the analysis of heart rate [4, 5]. Cardiointervalometry (chronocardiointervalometry) - a method of registration of sinus heart rate, followed by mathematical analysis of its structure. Cardiointervalogram is a serial number 100 cardio cycles recorded in the second standard lead in real time. R-R interval is the unit of measurement cardiointervalogram. Cardiointervalography as the dynamic range of the values of 
the duration of the cardiac cycle, can be viewed as a random process and hence is characterized by a distribution function. The distribution of $R-R$ intervals are stored the information on the status of human vital systems $[7,8]$.

Application of mathematical methods in the analysis of heart rate has more than half-century history. One of the most interesting approaches is entropy methods that are most effective in the case of the need to assess the body's response to any external influence. For this purpose, the author analyzes the frequency spectra rhythm gram as input during the wedge orthostatic test, allows displaying the potential of child functional systems.

To assess the parameters of a trend change, it was used an approach based on the use of entropy method of modeling complex systems. As noted above, the choice is motivated by the fact that one of the important characteristics of the «living» is «principle of disequilibrium», and no equilibrium processes in a closed system accompanied by a change of the entropy [1-3]. In this case, of the entropy values are determined for $\mathrm{CP}$ source (background) state $\left(H_{0}\right)$ and after exposure to $\left(H_{1}\right)$ according to the following formulas:

$$
H_{0}=-\sum_{i=1}^{n} P_{i} \cdot \log _{2} P_{i}
$$

Where $P_{i}-$ probability of getting $R-R$ interval in the corresponding class of the histogram.

$$
\begin{gathered}
H_{1}=-\sum_{i=1}^{n} P_{b(i)} \cdot \log _{2} P_{b(i)}, \\
P_{b(i)}=\frac{P_{i} \cdot p_{i}}{\sum_{r=1}^{n} P_{r} \cdot p_{r}}
\end{gathered}
$$

Where $P_{i}$ - probability of getting $R-R$ interval in the corresponding class of the histogram before and $p_{i}-$ after the exposure; value semantic information estimated by the formula:

$$
I_{\text {sem }}=-\sum_{i=1}^{n} \log _{2} \frac{P_{b(i)}}{P_{0(i)}}
$$

Where $P_{0(i)}$ - the probability of a discharge to the impact of the histogram; $n$ - the number of bits of the histogram.

To solve the problem of calculating the probabilities $P_{i}, p_{i}$, $P_{0(i)}$, in the formulas (1-4) is used method of constructing a histogram of values of the dynamic series Cardiointervalogram. Thus the following notation: Let $[a ; b]$, have a range of values of change $R-R_{i}$, and $k$ determines the number of ranks which covering this range, thus the sampling interval is calculated by the formula:

$$
W=\frac{b-a}{k},
$$

$k$ must be set in such a way that following inequality: $W \geq 0.05$ (this condition derived from the nature of functioning of biological objects and which is most commonly used when describing and modeling). Determine a sequence of numbers $K+2$ number $\left\{N_{i}\right\}$, where $i=0 \ldots k+1$ :

1) if then $N_{0}=N_{0}+1$;

2) if $a<R-R_{i} \leq b$, then let $J=\frac{R-R_{i}-a}{W}$ and select greatest integer less than or equal $J, N_{i}=N_{i}+1$;

$3)$ if $R-R_{i}>b$, then $N_{k+1}=N_{k+1}+1$.

In [5] shows the result of applying this approach to study the features of $\mathrm{CP}$ children in the early neonatal period. As an external force used wedge orthostatic test. Based on an analysis of indicators $H_{0}, H_{1}$ and $I_{\text {sem }}$ were identified 16 basic types of dynamics entropy of heart rate, among which are 5 main types of universal reaction has been allocated in the end $[6,7]$.

\section{BASED ON INFORMATION ENTROPY CRITERIA}

Information - entropy method is an example of so-called external describe the state of complex systems. This approach has its origin from the ideas expressed by Aristotle, according to which the importance of the whole above the importance of its components. It is known that the entropy of living systems - is a measure of the uncertainty distribution of states of biological systems.

With equal probability that the system is completely disorganized etc. At every moment in time, it can go to any state (in this case, the system has the maximum entropy). Increase of order means an increase in the relationship between the factors determining the behavior of the system, which leads to predictability of its behavior. According to some authors [2, 6], an increase of entropy in the transition from normality to pathology resist adaptation mechanisms. General adaptation syndrome mobilizes the body's functional reserves. Activates and labializations functional systems, which leads to a decrease in entropy. Overvoltage and depletion of regulatory mechanisms is accompanied by an increase in entropy, which, in particular, appears to reduce the coherence of the functional system, the deterioration of their synchronization.

The entropy of a living system can be determined with respect to distribution of any structural or functional characteristics. An important characteristic of living systems is the conditional entropy, the uncertainty characterizing the 
distribution of biological systems are relatively well-known (reference) distribution.

As a reference system parameters for biological systems can serve a variety of factors, first of all the variables of the environment of the system. A measure of conditional entropy can be used to assess the evolution of living systems in time. In this case, the probability distribution of the adoption of the reference system is its state at some point in time [8]. In addition, if the number of states at the same time remain unchanged, then the conditional entropy of the current distribution $P_{1}$ relative to a reference distribution $P_{2}$ is defined as:

$$
H\left(\frac{P_{1}}{P_{2}}\right)=\sum_{i=1}^{n} P_{1}\left(x_{i}\right) \cdot \log \left[\frac{P_{1}\left(x_{i}\right)}{P_{2}\left(x_{i}\right)}\right] .
$$

Overall of entropy change is accompanied by all the processes of vital life activity and serve as one of the characteristics in the analysis of biological laws. Authors for evaluating of entropy was introduced integrated indicator allowing to assess changes in Biosystems conditions at any time [8].

Any integral indicator summarizes all local rates in some area (time, spatial, situational). In turn, local estimates - is any quantitative assessments that are made on the basis of measurements in a single point - whether any moment in time, a point in the geometric point in space or status space. Formation of this situation can be written as follows. Let the set of points, which is considered our assessment, there is a $Z$ - set of elements $z_{1}, z_{2}, \ldots, z_{n}: Z=\left\{z_{i}\right\}$.

Local assessment of $h$, measured on each element, there is $h\left(z_{i}\right)$. If the set $Z$ is continuous, integral estimation can be written as an integral $H=\int_{z} h(Z) d Z$. Estimates of $h$ at the adjacent points may reflect temporary changes in performance (if $z_{i}=\mathrm{t}$-times). Then $H=\int_{0}^{T} h(t) d t .[0, \mathrm{~T}]-$ the time interval over which the observations were made. In our case, as the local estimates, $\left\{X_{1}, X_{2}, \ldots, X_{n}\right\}$, use the variables that characterize the functional state of the human body.

Introduced in [8] an integral indicator is based on the following assumptions. Entropy biological object, can be defined as follows:

$$
H=-\sum_{i=1}^{m} \sum_{j=1}^{n} P_{i j} \cdot \ln P_{i j}
$$

where $n$ - number of variables that characterize the status of the object; $m$ - number of possible status; $P_{i j}-$ the probability of the $i$-th state of biological object at the value of the variable $X_{j}$ state.

These following relations must obtain: $\sum_{i=1}^{m} P_{i j}=1, j=1, n$. The probability $P_{i j}$ (in the case of the normal distribution) as follows:

$$
P_{i j}=P(|X-a|<d)=2 \varphi\left(\frac{d}{s}\right)-1,
$$

where $a$ - the expectation of feature $x_{j} ; \delta$ - the value of the current value of deviation $x_{j}$ from $a ; \sigma-$ the dispersion of feature $x_{j} ; F$-standardized normal distribution function.

All possible status of a Biosystemscan be grouped into two major groups: norm and pathology. In this case, $m=2$, and the values of the corresponding probabilities $P_{i j}$ characterize the degree of deviation $x_{j}$ from values of the variables $X_{j}$ «normal range», where $P_{i j}$ - the probability of «pathology» of state for the corresponding variable $X_{j}$. It is known that the dynamics of the entropy can be judged on the changing state of the Biosystems.

Entropy methods underlying the modeling of complex systems is closely linked to the use of different information criteria. In this study, to evaluate the degree of deviation from the status of the organism level of the normal functioning of the criterion based on Kullback information measure was used. In this case, the approach proposed by N.V.Bokuchaevoy and G.V.Mamasahlisovym, namely, consideration of the information measures as a measure of Kullback preference behavior biological object:

$$
I(t)=\int_{t} P_{0}(x) \ln \frac{P_{0}(x)}{P(x, t)} d x,
$$

where $P_{0}(x)-$ probability density of the current «equilibrium» state; $P(x, t)$ - the probability density of finding an object in the state $S_{i}(i \in\{1 \ldots m\})$ during the time interval $t$, at which the observations were made; $x(t)=\left(x_{1}, \ldots, x_{n}, t\right)-$ the characteristic signs describing the status of the object.

This criterion allows us to estimate the degree of deviation of the current state of the object from the "preferred» (or reference) state, taking as a «preferred» state of the object state in which the values of all state variables are equal to average values of the physiological norm (for homogeneous groups surveyed).We introduce as a criterion for assessing the current state of the object the following expression: 


$$
I=\sum_{j=1}^{n} P_{0 j} \operatorname{Ln} \frac{P_{0 j}}{P_{1 j}}
$$

where $P_{0 j}-$ apriori probability that characterizes the «preferred» the probability of an object's state; $P_{1 j}$ posteriori probability. The probability that the value of the characteristic $X$ corresponds to the «norm». In the expression (11), the probability $P_{0 j}=1$, since, as a «preferred» state of the object we have taken a state in which deviation $\delta=0$, hence:

$$
P_{0 j}=1-\left[2 F\left(\frac{0}{\sigma}\right)-1\right]=2-2 F(0)=2-2 \cdot 05=1 .
$$

By substituting the value obtained in (5), we obtain

$$
I=\frac{1}{n} \sum_{j=1}^{n} \frac{\ln 1}{P_{j}}
$$

In the case where all the values of the characteristic features $x_{j}(j=\overline{1, n})$ are equal specify to average values of the respective given characteristics, the value of $I$ will be zero:

$$
I=\frac{1}{n} \sum_{j=1}^{n} \ln \frac{1}{1}=\frac{n \ln 1}{n}=0 .
$$

As it is known, the internal state of the biological system at any given time is a vector whose components correspond to the numerical values of the variables selected to describe the system.

The use of any of the integral criterion for assessing the state of the object assumes the existence of thresholds above which corresponds to the transition to a new state. As this value was set to $\left(I_{k r}\right)$ index $I$, corresponding to the case when all the variables taken into account $x_{j}\{j=1, \ldots, n\}$ state «normal» and state «athology» are equally probable, i.e. The system with equal probabilities completely disorganized and any time it can go to any state (in this case, the system has the maximum entropy) that is in accordance with formula (12):

$$
I_{k r}=\frac{1}{n} \sum_{j=1}^{n} \ln \frac{1}{0,5}=\frac{n \ln 2}{n}=0,69
$$

From a practical point of view, it is extremely important to distinguish between changes that occur within a single state from the transition to the new functional state. In [8] we introduced three critical levels: «fatigue», «stress» and «collapse».

In this case, the less value of I, the nearer to the status of the object «preferred» (in the ideal case, $I=0$ ). The value of $\left(I_{k r}\right)$ is used as a criterion $I$ threshold value corresponding to the case when all the indicators taken into account the state of the biosystem «norm» and «voltage» are equally probable. In this case $I_{k r}=0.69$.

The increase in $I$ (at $I<I_{1}$ ) will correspond to the transition of the Biosystems from the «norm» to «fatigue». A further increase in $I$ (if $I>I_{2}$ ) will correspond to the transition from «fatigue» to «stress» and then to «disruption» [3]. For qualitative assessment of changes in state of the organism in a certain period, introduced the function $I(t)$, representing the value of the integral index (9) in different (current) times. The function $I(t)$ can be considered as a transition state function. One advantage of this function is expected that it is not given once but permanently (such as in a fairly widespread «automaton» approach to describe biological systems).For quantitative estimation of deviation variables $X_{j}$ state of the normal level using the integral index of the form [3]:

$$
S=\frac{S_{1}}{S_{0}}
$$

Where (then $\left.I(t)>I_{k r}\right)$

$$
S_{0}=\int_{0}^{t} I(t) d t ; \quad S_{1}=\int_{0}^{t} I(t) \operatorname{Sign}\left(I(t)-I_{\kappa p}\right) d t
$$

In the case where value of $I(t)$ will not exceed the $I_{k r}$ throughout the time of study entered integral index is zero. Due to the fact that the value of the entropy change depends on the number of state variables taken into account, we introduced normalized integral component [8]:

$$
Q=\frac{H_{1}}{H_{0}}
$$

Where $H$ - value of entropy; $H_{0}$ - the value of the entropy in the case where all the value of the variable state $x(j)$ are equal to the corresponding mathematical expectation. The character of changing parameter $Q$ allows you to emphasize the so-called «at risk», i.e. trhe transitional state from normal to pathology (the state of «surge»).

In the transition state of the Biosystems from the norm to state «surge» the value of $\frac{H_{1}}{H_{0}}$ will increase from 1 to $Q_{\max }$. 
In the transition from the «surge» to the disease - reduced from $Q_{\max }$ to 1 .

Dependency can be obtained, apparently results from the following Entropy Biosystems $(\mathrm{H})$ can be written as: $H=H_{0}+\Delta H$, where the increment of entropy $\Delta H$ consists of the increment of entropy due to changes in the internal state of the system $\Delta H_{E x c h}$, i.e. $\Delta H=\Delta H_{I n}+\Delta H_{E x c h}$. Status of the current balance for open systems (and any biological system consider an open system) is determined by the following: $\Delta H(t)=0$, or $\Delta H_{I n}(t)=\Delta H_{E x c h}(t)$.

Moreover, the impaired balance associated either with a change in the internal state, or with a deviation of the exchange processes. Apparently, at risk starts to happen violation of the exchange process which causes a decrease in the production of negative entropy $\left(\Delta H_{E x c h}\right)$ and an increase in the total increase of entropy ( $\Delta H$ ). In the disease area is a violation of the internal condition that causes a slowdown in growth of positive entropy $\left(\Delta H_{I n}\right)$, reducing the overall gain of the entropy $H$ and the reduction coefficient $Q$.

\section{CONCLUSION}

The proposed information entropy criteria allow obtaining qualitatively new results in solving various applied tasks like for example:

- Assess the state of adaptation of Petroleum and loggers under Watches;

- Assessing the body condition based on the analysis results of functional tests;

- Diagnostic newborn state in the early neonatal period;

- Tracking the dynamics of the human body condition in the postoperative period;

- Psychophysiological assessment of the level of readiness of students to professional work.

The validity of this approach to assess the psychological and psychophysiological states stems from the possibility of considering the psychological state of the person from the position of synergy. Synergetic concept allows to consider the structure of the individual in the process of socialization as a non-equilibrium, unstable self-organizing system has a certain margin of stability Dynamic changes in the course of socialization, lead to the emergence of new formations of personality characteristics and qualities of the different degree of stability.

With the system approach, specializing personality is seen as a large component of the system - society. In this system, society functions are a particular influence on process of formation the personality of its structural entities. Due to regular function of society is achieved by adaptation effect, i.e. bringing the characteristics of the subject to the requirements of professional media activities. Changes are made possible by integrating the structure of personality function, which in the space of energy and information - Time coordinates associates, on the one hand, the features the external environment, which includes the social environment, professional activity, on the other - personal formation, forming the corresponding structures.

Functional structures of personal educational research reveals three-level functional structures, characterized by the specificity of functions in segments of actual, current and long socialization. System consideration of functional structures with a common position allows to set the main basic features of personality structures in the process of socialization: a regulatory function, reflecting function, integration of differentiation, as well as highlight the features of the structural organization of professionally significant personal structures. In accordance with these bases, the psychological state of the person in the process of socialization may have a different energy level, which is reflected in the qualitative specifics of personality structures. For a reference, point (0) may be taken with respect to the equilibrium state - state average or optimum psychological state in the process of socialization.

States associated with increased or decreased mental activity, characterized by a correspondingly high or low relative to the psychological state of the midpoint can be attributed to non-equilibrium states. In general, all the psychological state of the person in the process of socialization may be calibrated higher or lower than the zero point.

It is obvious that non-equilibrium psychological state of the person in the process of socialization of different modalities and intensity has a specific quality is reflected in the composition, structure, functions, determination of the formation of one or another of professionally significant personal structures. Equilibrium psychological state of the person is the foundation of an adequate professional activity. Actualization of no equilibrium psychological state of the person in the process of socialization can cause inadequate professional work, communication.

From the standpoint of system analysis and concepts of self-organizing nonequilibrium psychological state of the person in the process of socialization is a functional structure, formed in violation of symmetry between the individual and the social professional environment. Violations of symmetry arise from the processes caused by the introduction of information and energy flow in an open system, which is for society and the individual. As a result, due to selforganization, formed a new functional state of the system non-equilibrium state.

\section{REFERENCES}

[1]. Prangishvili I.V. Jentropijnye i drugie sistemnye zakonomernosti: Voprosy upravlenija slozhnymi sistemami / I.V. Prangishvili; In-t problem upravlenija im. V.A. Trapeznikova. - M.: Nauka, 2003. -428 p. 
[2]. Prigozhin I., Stengers I. Vremja. Haos. Kvant - M. : Jeditorial URSS, 2000. - $239 \mathrm{p}$.

[3]. Rotov A.V., Medvedev M.A., Pekker Ja.S., Berestneva O.G. Adaptacionnye harakteristiki cheloveka. - Tomsk: Izd-vo Tomskogo gos. un-ta, 1997. $-137 \mathrm{p}$.

[4]. Baevskij R.M., Ivanov G.G., Chirejkin V.V. i dr. Analiz variabel'nosti serdechnogo ritma pri ispol'zovanii razlichnyh kardiograficheskih sistem (metodicheskie rekomendacii) / /Vestnik aritmologii, 2001. №24.-pp. 65-87.

[5]. Kojchubekov B.K. Jentropijnye pokazateli kardiointervalogrammy v razlichnyh vozrastnyh gruppah // Izvestija NAN RK, Serija biologicheskaja i medicinskaja, 2006. - №3 (255) . - pp. 56-59.

[6]. Gerget O.M., Berestneva O.G., Pekker Ja.S. Avtomatizirovannaja informacionnaja sistema ocenki adaptacii razvivajushhihsja sistem. Problemy informatiki, 2011. - № 2. - S. 76-82.

[7]. Simulation and evaluation of biological systems adaptive capabilities / Berestneva, O.G. Pekker, J.S. (2014) Proceedings of 2014 International Conference on Mechanical Engineering, Automation and Control Systems, MEACS p. 201

[8]. Berestneva O.G., Pekker Y.S., Murzina S.S. Entropy methids in analysis of biological systems.// Bulletin of Siberian Medicine 2014;13(4):15-20. (In Russ.) DOI:10.20538/1682-0363-2014-4-15-20 